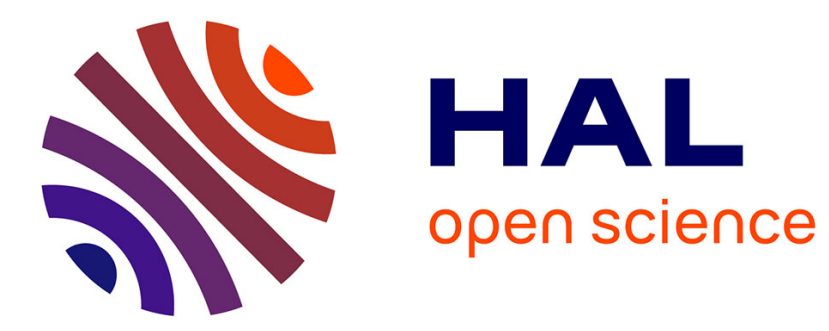

\title{
Spectral properties of chaotic processes
}

Bernard Bercu, Clémentine Prieur

\section{To cite this version:}

Bernard Bercu, Clémentine Prieur. Spectral properties of chaotic processes. Stochastics and Dynamics, 2006, 6 (3), pp.355-371. hal-00023715

\section{HAL Id: hal-00023715 https://hal.science/hal-00023715}

Submitted on 4 May 2006

HAL is a multi-disciplinary open access archive for the deposit and dissemination of scientific research documents, whether they are published or not. The documents may come from teaching and research institutions in France or abroad, or from public or private research centers.
L'archive ouverte pluridisciplinaire HAL, est destinée au dépôt et à la diffusion de documents scientifiques de niveau recherche, publiés ou non, émanant des établissements d'enseignement et de recherche français ou étrangers, des laboratoires publics ou privés. 


\title{
Spectral properties of chaotic processes
}

\author{
Bernard Bercu* and Clémentine Prieur ${ }^{\dagger}$
}

May 4, 2006

\begin{abstract}
We investigate the spectral asymptotic properties of the stationary dynamical system $\xi_{t}=\varphi\left(T^{t}\left(X_{0}\right)\right)$. This process is given by the iterations of a piecewise expanding map $T$ of the interval $[0,1]$, invariant for an ergodic probability $\mu$. The initial state $X_{0}$ is distributed over $[0,1]$ according to $\mu$ and $\varphi$ is a function taking values in $\mathbb{R}$. We establish a strong law of large numbers and a central limit theorem for the integrated periodogram as well as for Fourier transforms associated with $\left(\xi_{t}\right)$. Several examples of expanding maps $T$ are also provided.
\end{abstract}

AMS subject classifications. Primay 37A50; secondary 60F05, 60F15.

Key words and phrases. Integrated periodogram, Fourier transforms, strong law, central limit theorem, dynamical systems.

\section{Introduction.}

Over the last decade, the statistical properties of chaotic processes have been deeply investigated in order to modalize complex systems [1], [2], [15]. More precisely, chaotic dynamical systems such as expanding maps of the interval have been suggested to capture the complexity of packet traffic [15] or to analyse the measurements of communication traffic from a wide variety of sources [2]. Chaos is the phenomenon by which low order nonlinear dynamical systems exhibit complex, seemingly random behavior. One can notice that trajectories of chaotic systems are very often fractal in nature, hence they can be used as convenient generators of fractal structures. In this paper, we shall focus our attention on the strictly stationary dynamical system given, for all $t \in \mathbb{N}$, by

$$
\xi_{t}=\varphi\left(T^{t}\left(X_{0}\right)\right)=\varphi\left(X_{t}\right)
$$

*Institut de Mathématiques, Laboratoire de Statistique et Probabilités, Université Paul Sabatier, Toulouse Cedex, France. E-mail: bernard.bercu@math.ups-tlse.fr

${ }^{\dagger}$ INSA Toulouse et Institut de Mathématiques, Laboratoire de Statistique et Probabilités, Toulouse Cedex, France. E-mail: clementine.prieur@insa-toulouse.fr 
where $T$ is a piecewise expanding map [6] of the interval $[0,1]$, invariant for an ergodic probability $\mu$. The initial state $X_{0}$ is distributed over $[0,1]$ according to $\mu$ and $\varphi$ is a function from $[0,1]$ to $\mathbb{R}$. Under suitable assumptions on $T$ and $\varphi$, we shall establish a strong law of large numbers and a central limit theorem for the integrated periodogram associated with $\left(\xi_{t}\right)$. We shall also prove similar results for Fourier transforms of $\left(\xi_{t}\right)$. We will apply our results to several parametric Lasota-Yorke maps [18] which are piecewise expanding maps of the interval $[0,1]$. It is well known $[6,8,17,19,20]$ that such maps admit a unique absolutely continuous invariant measure. One can find few papers dealing with the problem of the nonparametric estimation of the invariant density [7, 9, 24]. Our purpose is now to analyse the spectral asymptotic properties of such chaotic processes. We shall assume that the process $\left(\xi_{t}\right)$ is zero mean and we denote by $(\gamma(t))$ its covariogram defined, for all $t \geq 0$, by

$$
\gamma(t)=\mathbb{E}_{\mu}\left[\xi_{0} \xi_{t}\right]
$$

The spectral density of $\left(\xi_{t}\right)$ is given, for all $\lambda$ in the torus $\mathbb{T}=[-\pi, \pi[$, by

$$
f(\lambda)=\frac{1}{2 \pi} \sum_{t \in \mathbb{Z}} \gamma(|t|) e^{-i t \lambda} .
$$

If the maps $\varphi$ and $T$ are both continuous and $(\gamma(t))$ goes to zero at a polynomial rate of order $>2$, Lopes and Lopes [21] have proven the convergence in distribution sense of the empirical periodogram to the spectral density $f$. One can observe that the periodogram is evaluated on the discrete Fourier frequencies. More recently, Chazottes, Collet and Schmitt [7] studied the convergence in $\mathbb{L}^{2}$ of the empirical spectral distribution for a wide class of dynamical systems, including piecewise expanding maps of the interval $[0,1]$ satisfying Devroye's inequality.

Our approach is totally different. We first propose central limit theorem for the integrated periodogram of $\left(\xi_{t}\right)$, in the spirit of the original work of Rosenblatt [25]. This result only holds under several conditions on the stationary process and on its covariogram. We propose projective criteria, expressed with projective coefficients in the style of Gordin, under which assumptions for the central limit theorem are satisfied. We next introduce the time reversal process $\left(Y_{t}\right)$ associated with the underlying process $\left(X_{t}\right)$. The process $\left(Y_{t}\right)$ is a Markov chain [4]. One can check that $\left(X_{0}, X_{1}, \cdots, X_{t}\right)$ shares the same distribution as $\left(Y_{t}, Y_{t-1}, \cdots, Y_{0}\right)$. Via Markov arguments, we show that the projective criteria are fulfilled for our reversed process $\left(\varphi\left(Y_{t}\right)\right)$. Finally, by use of similar techniques, we deduce from [29] the asymptotic behavior of Fourier transforms of $\left(\xi_{t}\right)$ defined, for a given real-valued function $g$ and for all $\theta \in \mathbb{R}$, by

$$
S_{n}(\theta)=\sum_{t=1}^{n} g\left(\xi_{t}\right) e^{i t \theta}
$$


The paper is organized as follows. In Section 2, we give the central limit theorem for the integrated periodogram of $\left(\xi_{t}\right)$. Section 3 is devoted to the time reversal Markov chain $\left(Y_{t}\right)$ associated with $\left(X_{t}\right)$. Coefficients of dependence, very useful to measure the dependence structure of the underlying process $\left(X_{t}\right)$, are given in Section 4. We also propose projective criteria under which the assumptions of the central limit theorem are satisfied. By use of $\left(Y_{t}\right)$, we show that the projective criteria are fulfilled so that we get the central limit theorem for the integrated periodogram of $\left(\xi_{t}\right)$. Several examples of expanding maps $T$ whose reversed process satisfies the projective criteria are provided in Section 5. Section 6 is devoted to the asymptotic results concerning the Fourier transforms of $\left(\xi_{t}\right)$ while Section 7 deals with the nonstationary case. Finally, all the technical proofs are postponed to Section 8.

\section{Integrated periodogram}

We shall now define the integrated periodogram associated with $\left(\xi_{t}\right)$ given by (1) and investigate its asymptotic properties. Assume in all the sequel that $\mathbb{E}_{\mu}\left[\xi_{0}^{4}\right]$ is finite. The fourth cumulants of $\left(\xi_{t}\right)$ are given, for all $(r, s, t) \in \mathbb{Z}^{3}$, by

$$
\kappa(r, s, t)=\mathbb{E}_{\mu}\left[\xi_{0} \xi_{r} \xi_{s} \xi_{t}\right]-\mathbb{E}_{\mu}\left[\xi_{0} \xi_{r}\right] \mathbb{E}_{\mu}\left[\xi_{s} \xi_{t}\right]-\mathbb{E}_{\mu}\left[\xi_{0} \xi_{s}\right] \mathbb{E}_{\mu}\left[\xi_{r} \xi_{t}\right]-\mathbb{E}_{\mu}\left[\xi_{0} \xi_{t}\right] \mathbb{E}_{\mu}\left[\xi_{r} \xi_{s}\right]
$$

By stationarity of the process $\left(\xi_{t}\right)$, those cumulants may be defined over $\mathbb{Z}^{3}$. It is natural [25] to assume that

$$
\gamma=\sum_{t \in \mathbb{N}} \gamma(t)^{2}<+\infty \quad \text { and } \quad \kappa=\sum_{(r, s, t) \in \mathbb{Z}^{3}}|\kappa(r, s, t)|<+\infty .
$$

The empirical periodogram associated with $\left(\xi_{t}\right)$ is defined, for all $\lambda \in \mathbb{T}$, by

$$
I_{n}(\lambda)=\frac{1}{2 \pi n}\left|\sum_{t=1}^{n} \xi_{t} e^{-i t \lambda}\right|^{2}
$$

Let $\left(\gamma_{n}(t)\right)$ be the empirical covariances given, for all $0 \leq t \leq n-1$, by

$$
\gamma_{n}(t)=\frac{1}{n} \sum_{k=1}^{n-t} \xi_{k} \xi_{t+k}
$$

and $\gamma_{n}(t)=0$ if $t \geq n$. One can easily see from (5) that, for all $\lambda \in \mathbb{T}$

$$
I_{n}(\lambda)=\frac{1}{2 \pi} \sum_{t \in \mathbb{Z}} \gamma_{n}(|t|) e^{-i t \lambda}
$$

It is well known that $I_{n}(\lambda)$ is not a good estimator of $f(\lambda)$. It is more appropriate to make use of the integrated periodogram

$$
\mathcal{I}_{n}(g)=\int_{-\pi}^{\pi} g(\lambda) I_{n}(\lambda) d \lambda
$$


where $g$ belongs to the set of functions

$$
\mathcal{G}=\left\{g: \mathbb{T} \rightarrow \mathbb{R}, 2 \pi \text {-periodic and continuous with } g \in \mathbb{L}^{2}(\mathbb{T})\right\} .
$$

We shall prove the almost sure convergence of $\mathcal{I}_{n}(g)$ to

$$
\mathcal{I}(g)=\int_{-\pi}^{\pi} g(\lambda) f(\lambda) d \lambda
$$

together with a central limit theorem which makes use of the bispectral density function

$$
f_{4}(\lambda, \mu, \nu)=\frac{1}{(2 \pi)^{3}} \sum_{(r, s, t) \in \mathbb{Z}^{3}} \kappa(r, s, t) e^{-i(r \lambda+s \mu+t \nu)} .
$$

Theorem 1 For all $t \in \mathbb{N}$,

$$
\lim _{n \rightarrow \infty} \gamma_{n}(t)=\gamma(t) \quad \text { a.s. }
$$

In addition, assume that (4) holds and, for all $t \in \mathbb{N}$,

$$
\sum_{s=1}^{\infty}\left(\mathbb{E}\left[\left(\mathbb{E}\left[\xi_{0} \xi_{s} \mid \xi_{t+s}\right]-\gamma(t)\right)^{2}\right]\right)^{1 / 2}<\infty
$$

and

$$
\sigma^{2}(t)=\mathbb{E}\left[\left(\xi_{0} \xi_{t}-\gamma(t)\right)^{2}\right]+2 \sum_{s=1}^{\infty} \mathbb{E}\left[\left(\xi_{s} \xi_{t+s}-\gamma(t)\right)\left(\xi_{0} \xi_{t}-\gamma(t)\right)\right]>0 .
$$

Then, for all $t \in \mathbb{N}$,

$$
\sqrt{n}\left(\gamma_{n}(t)-\gamma(t)\right) \stackrel{\mathcal{L}}{\longrightarrow} \mathcal{N}\left(0, \sigma^{2}(t)\right) .
$$

Furthermore, for all $s, t \in \mathbb{N}$, set

$$
\sigma(s, t)=\mathbb{E}\left[\left(\xi_{0} \xi_{s}-\gamma(s)\right)\left(\xi_{0} \xi_{t}-\gamma(t)\right)\right]+2 \sum_{r=1}^{\infty} \mathbb{E}\left[\left(\xi_{r} \xi_{t+r}-\gamma(t)\right)\left(\xi_{0} \xi_{s}-\gamma(s)\right)\right] .
$$

Then, for all $d \geq 1$ and for arbitrary distinct integers $t_{1}, t_{2}, \cdots, t_{d} \in \mathbb{N}$, we have

$$
\left\{\sqrt{n}\left(\gamma_{n}\left(t_{i}\right)-\gamma\left(t_{i}\right)\right)\right\}_{1 \leq i \leq d} \stackrel{\mathcal{L}}{\longrightarrow} \mathcal{N}_{d}(0, \Gamma)
$$

where $\Gamma$ is the positive definite covariance matrix given by $\Gamma=\left(\sigma\left(t_{i}, t_{j}\right)\right)_{1 \leq i, j \leq d}$.

Theorem 2 For all $g \in \mathcal{G}$,

$$
\lim _{n \rightarrow \infty} \mathcal{I}_{n}(g)=\mathcal{I}(g) \quad \text { a.s. }
$$

In addition, under assumptions (4), (8) and (9), we have the finite dimensional convergence of $\left\{\sqrt{n}\left(\mathcal{I}_{n}(g)-\mathcal{I}(g)\right), g \in \mathcal{G}\right\}$ to the zero mean Gaussian process $\{Z(g), g \in \mathcal{G}\}$ with covariance given, for all $g_{1}, g_{2} \in \mathcal{G}$ by

$$
\Gamma\left(g_{1}, g_{2}\right)=4 \pi \int_{-\pi}^{\pi} g_{1}(\lambda) g_{2}(\lambda) f^{2}(\lambda) d \lambda+2 \pi \int_{-\pi}^{\pi} \int_{-\pi}^{\pi} g_{1}(\lambda) g_{2}(\mu) f_{4}(\lambda,-\mu, \mu) d \lambda d \mu .
$$

Proof. The proof of Theorems 1 and 2 are postponed to Section 8 . 


\section{The associated Markov chain}

For the process $\left(\xi_{t}\right)$, the randomness only enters when setting the initial state. The analogy with classical process is therefore clearer when considering the reversed process, for which randomness enters progressively at each step. Starting from this remark of Barbour \& al. [4], we introduce the time reversal process $\left(Y_{t}\right)$ associated with the underlying process $\left(X_{t}\right)$ given by $(1)$. First of all, let $\mathcal{L}$ be the operator from $\mathbb{L}^{1}([0,1])$ to $\mathbb{L}^{1}([0,1])$ given, for all functions $h \in \mathbb{L}^{1}([0,1])$ and $k \in \mathbb{L}^{\infty}([0,1])$, by the identity

$$
\int_{0}^{1} \mathcal{L}(h)(x) k(x) d x=\int_{0}^{1} h(x) k(T(x)) d x .
$$

The operator $\mathcal{L}$ is called the Perron-Frobenius operator of $T$. Assume that the probability distribution $\mu$ is absolutely continuous with respect to the lebesgue measure. Denote by $f_{\mu}$ the density function associated with $\mu$. Let $I^{*} \subset[0,1]$ be the support of $\mu$ and choose a version of $f_{\mu}$ such that $f_{\mu}>0$ on $I^{*}$ and $f_{\mu}=0$ otherwise. One can observe that it is possible to choose $\mathcal{L}$ such that $\mathcal{L}\left(f_{\mu} h\right)(x)=\mathcal{L}\left(f_{\mu} h\right)(x) \mathbb{I}_{f_{\mu}(x)>0}$. Let $K$ be the Markov kernel associated to $T$ given, for all $x \in[0,1]$, by

$$
K(h)(x)=\frac{\mathcal{L}\left(f_{\mu} h\right)(x)}{f_{\mu}(x)} \mathbb{I}_{f_{\mu}(x)>0}+\mu(h) \mathbb{I}_{f_{\mu}(x)=0} .
$$

The time reversal process $\left(Y_{t}\right)$ associated with $\left(X_{t}\right)$ is a stationary Markov chain with invariant distribution $\mu$ and transition kernel $K$. It is easy to check [4] that $\left(X_{0}, X_{1}, \cdots, X_{t}\right)$ shares the same distribution as $\left(Y_{t}, Y_{t-1}, \cdots, Y_{0}\right)$. Hence, to prove Theorem 1, we study the asymptotic behavior of

$$
\sqrt{n}\left(\frac{1}{n} \sum_{k=1}^{n-t} \varphi\left(Y_{k}\right) \varphi\left(Y_{t+k}\right)-\gamma(t)\right) .
$$

Consequently, it is necessary to go further in the study of the reversed process $\left(Y_{t}\right)$. Denote by $B V([0,1])$ the set of functions

$$
B V([0,1])=\left\{h:[0,1] \rightarrow \mathbb{R} \text { such that } h \in \mathbb{L}^{1}([0,1]),\|D h\|<+\infty\right\}
$$

where $\|D h\|=|D h|([0,1])$ stands for the total variation of the distributional derivative of $h$ on $[0,1]$. Of course, if $h$ is absolutely continuous, $D h$ is a function which coincides with the pointwise derivative $h^{\prime}$ of $h$. The set $B V([0,1])$ is a Banach space endowed with the norm $\|h\|_{v}=\|h\|_{1}+\|D h\|$. In many interesting cases, the spectral analysis of the Perron-Frobenius operator $\mathcal{L}$ in the Banach space $B V([0,1])$ can be achieved by use of Ionescu-Tulcea and Marinescu theorem [6], [18]. Assume that 1 is a simple eigenvalue of $\mathcal{L}$ and that the rest of the spectrum is contained in a closed disk of radius strictly smaller than one. Then, one can find an unique $T$-invariant absolutely continuous probability distribution $\mu$ whose density function $f_{\mu}$ belongs 
to $B V([0,1])$, such that all the powers of the Kernel $K$ can be decomposed for all $x \in[0,1]$, as

$$
K^{n}(h)(x)=\frac{\Psi^{n}\left(f_{\mu} h\right)(x)}{f_{\mu}(x)} \mathbb{I}_{f_{\mu}(x)>0}+\mu(h) \mathbb{I}_{f_{\mu}(x)=0}
$$

with $\Psi\left(f_{\mu}\right)=0$ and

$$
\left\|\Psi^{n}(h)\right\|_{v} \leq c \rho^{n}\|h\|_{v}
$$

for some $0<\rho<1$ and $c>0$. In addition, assume that

$$
d=\left\|\frac{1}{f_{\mu}} \mathbb{I}_{f_{\mu}>0}\right\|_{v}<\infty
$$

It was proven by Dedecker and Prieur [10] that, for any functions $h \in B V([0,1])$ and $k \in \mathbb{L}^{1}([0,1])$,

$$
\left|\operatorname{Cov}\left(h\left(X_{0}\right), k\left(X_{n}\right)\right)\right| \leq a_{n}\left\|k\left(X_{n}\right)\right\|_{1}\|h\|_{v}
$$

where $a_{n}=\alpha \rho^{n}$ with $\alpha=2 c d\left(\left\|D f_{\mu}\right\|+1\right)$. We refer to the paper of Broise [6] for examples of dynamics $T$ satisfying assumption (15) and to the paper of Morita [23] for sufficient conditions implying assumption (16). Two examples of piecewise expanding maps are detailed in Section 5.

\section{Coefficients of dependence}

In order to check that the assumptions of Theorem 1 are satisfied, we introduce coefficients of dependence which allow us to measure the dependence structure of the reversed process $\left(\varphi\left(Y_{t}\right)\right)$. For dynamical systems, and in particular for piecewise expanding maps of the interval, such coefficients of dependence are closely related to covariance inequalities.

Definition 1 Let $\left(Z_{t}\right)$ be a stationary sequence of zero mean real-valued random variables. Let $\mathbb{F}=\left(\mathcal{F}_{t}\right)$ be the filtration given by $\mathcal{F}_{t}=\sigma\left(Z_{0}, Z_{1}, \cdots Z_{t}\right)$ and denote by $\mathbb{E}_{t}$ the conditional expectation with respect to $\mathcal{F}_{t}$. For any integers $0 \leq i<j$ and $k \geq 0$, let $\Gamma_{i, j, k}$ be the set of multiintegers $\left(t_{1}, t_{2}, \cdots t_{j}\right)$ such that $0 \leq t_{1} \leq \cdots \leq t_{i}$ and $t_{i}+k \leq t_{i+1} \leq \cdots \leq t_{j}$. For $m \in\{1,2\}$, set

$$
\theta_{i, j, m}(k)=\sup _{\left(t_{1}, \ldots, t_{j}\right) \in \Gamma_{i, j, k}} \| Z_{t_{1}} \cdots Z_{t_{i}} \mathbb{E}_{t_{i}}\left[Z_{t_{i+1}} \cdots Z_{t_{j}}-\mathbb{E}\left[Z_{t_{i+1}} \cdots Z_{t_{j}}\right] \|_{m}\right.
$$

In the case where $m=1$, these coefficients have been first introduced [11] to derive Esseen's mean central limit theorems for dependent sequences.

We now give projective criteria using these dependence coefficients under which the central limit theorem for the integrated periodogram of a stationary process with mean zero holds. 
Theorem 3 Assume that the reversed process $\left(\varphi\left(Y_{t}\right)\right)$ associated to the process $\left(\xi_{t}\right)$ given by (1) satisfies the three following conditions (a), (b) and (c). Then, (4) and (8) hold true for $\left(\xi_{t}\right)$.

(a) $\sum_{k=0}^{\infty} \theta_{1,2,1}(k)<+\infty$,

(b) For any $1 \leq i<j \leq 4, \sum_{k=0}^{\infty} k^{j-2} \theta_{i, j, 1}(k)<+\infty$,

(c) $\sum_{k=1}^{\infty} \theta_{0,2,2}(k)<+\infty$.

Proof. The proof of Theorem 3 is postponed to Section 8 .

Theorem 4 Assume (15) and (16) hold. Moreover, suppose that $\varphi$ is in $B V([0,1])$. Then, assumptions $(a),(b)$ and $(c)$ of Theorem 3 are satisfied.

Proof. The proof is given in Section 8 .

Remark. Under assumptions (15) and (16), we know from Theorems 3 and 4 that (4) and (8) hold true for $\left(\xi_{t}\right)$. But we didn't say anything about assumption (9) of Theorem 1. The case

$$
\sigma^{2}(t)=\mathbb{E}\left[\left(\xi_{0} \xi_{t}-\gamma(t)\right)^{2}\right]+2 \sum_{s=1}^{\infty} \mathbb{E}\left[\left(\xi_{s} \xi_{t+s}-\gamma(t)\right)\left(\xi_{0} \xi_{t}-\gamma(t)\right)\right]=0
$$

is a degenerate case. The result of Theorem 1 still holds but the limit is a Gaussian law with variance zero. Let us give a simple situation under which we can prove that we are not in the degenerate case. We know from [25, Chapter III] that the limiting variance in Theorem 1 satisfies

$$
\sigma^{2}(t)=\lim _{n \rightarrow+\infty} \frac{1}{n} \sum_{k=0}^{n} \sum_{l=0}^{n} \operatorname{Cov}\left(\xi_{k} \xi_{k+t}, \xi_{l} \xi_{l+t}\right) .
$$

Let us endow $B V([0,1])$ with the scalar product defined, for all $h, k$ in $B V([0,1])$, by

$$
<h, k>_{\mu}=\int_{0}^{1} h(x) k(x) f_{\mu}(x) d x .
$$

For all $x \in[0,1]$ and $t \geq 0$, set $h_{t}(x)=\varphi(x) \varphi\left(T^{t}(x)\right)-\gamma(t)$. Relation (19) can be rewritten as

$$
\sigma^{2}(t)=\lim _{n \rightarrow+\infty} \frac{1}{n} \sum_{k=0}^{n} \sum_{l=0}^{n}<h_{t}\left(T^{k}\right), h_{t}\left(T^{l}\right)>_{\mu}=\sum_{k \in \mathbb{Z}}<h_{t}\left(T^{k}\right), h_{t}>_{\mu} .
$$

Hence, it follows from Proposition 7.1 of [6] that, for a piecewise expanding map $T$, if the associated partition is countably infinite, and if assumption (16) is satisfied, then the limit $\sigma^{2}(t)$ is strictly positive as soon as the function $\varphi$ is not a constant. The proof relies on Ionescu-Tulcea and Marinescu Theorem on the spectral decomposition of the Perron-Frobenius operator $\mathcal{L}$ associated to $T$. 


\section{Piecewise expanding maps}

Several examples of expanding maps $T$ satisfying conditions (15) and (16) are given in [6, page 11]. We shall now focuse our attention on two specific transformations. On the one hand, for some integer $a \geq 2$ and for all $x \in[0,1]$, let

$$
T_{a}(x)=a x-[a x]
$$

This map is commonly called $a$-transformation. The invariant probability $\mu$ is the Lebesgue measure on $[0,1]$. A straightforward calculation leads to

$$
\mathbb{E}\left[X_{0}\right]=\frac{1}{2} \quad \text { and } \quad \operatorname{Var}\left(X_{0}\right)=\frac{1}{12}
$$

Consequently, for all $x \in[0,1]$, we choose $\varphi(x)=x-1 / 2$. It is not hard to see that for all $t \geq 0$

$$
\gamma(t)=\frac{1}{12 a^{t}}
$$

Hence, if we set

$$
\sigma^{2}=\frac{a^{2}-1}{12}
$$

we deduce via (3) that the spectral density associated with (20) is given, for all $\lambda \in \mathbb{T}$, by

$$
f(\lambda)=\frac{\sigma^{2}}{2 \pi\left(1+a^{2}-2 a \cos (\lambda)\right)} .
$$

We can estimate the unknown parameter $a$ by the Yule-Walker estimator

$$
\widehat{a}_{n}=\frac{\gamma_{n}(0)}{\gamma_{n}(1)}=\frac{\sum_{k=1}^{n} \xi_{k}^{2}}{\sum_{k=1}^{n-1} \xi_{k} \xi_{k+1}} .
$$

It immediately follows from Theorem 1 that $\widehat{a}_{n} \longrightarrow a$ a.s. Furthermore, we have the decomposition

$$
\sqrt{n}\left(\widehat{a}_{n}-a\right)=\frac{\sqrt{n}}{\gamma_{n}(1)}\left(\gamma_{n}(0)-\gamma(0)-\frac{\gamma(0)}{\gamma(1)}\left(\gamma_{n}(1)-\gamma(1)\right)\right) .
$$

Consequently, we infer from (11) together with Slutsky's lemma that

$$
\sqrt{n}\left(\widehat{a}_{n}-a\right) \stackrel{\mathcal{L}}{\longrightarrow} \mathcal{N}\left(0, \tau^{2}\right)
$$

where

$$
\tau^{2}=(12 a)^{2}\left(\sigma^{2}(0)+a^{2} \sigma^{2}(1)-2 a \sigma(0,1)\right) .
$$

On the other hand, consider the transformation given, for some $0<a<1$ and for all $x \in[0,1]$, by

$$
T_{a}(x)=\left\{\begin{array}{lll}
\frac{x}{a} & \text { if } & 0 \leq x<a \\
\frac{1-x}{1-a} & \text { if } & a \leq x<1
\end{array}\right.
$$


As before, the invariant probability $\mu$ is the Lebesgue measure on $[0,1]$ so that we also take, for all $x \in[0,1], \varphi(x)=x-1 / 2$. One can easily see [22, page 26] that for all $t \geq 0$

$$
\gamma(t)=\frac{1}{12}(2 a-1)^{t}
$$

If

$$
\sigma^{2}=\frac{a(1-a)}{6}
$$

the spectral density associated with (21) is given, for all $\lambda \in \mathbb{T}$, by

$$
f(\lambda)=\frac{\sigma^{2}}{2 \pi(1-2 a(1-a)+(2 a-1) \cos (\lambda))} .
$$

We can estimate the unknown parameter $a$ by the Yule-Walker estimator

$$
\widehat{a}_{n}=\frac{1}{2}\left(\frac{\gamma_{n}(1)}{\gamma_{n}(0)}+1\right)=\frac{1}{2}\left(\frac{\sum_{k=1}^{n-1} \xi_{k} \xi_{k+1}}{\sum_{k=1}^{n} \xi_{k}^{2}}+1\right) .
$$

We immediately deduce from Theorem 1 that $\widehat{a}_{n} \longrightarrow a$ a.s. In addition, one can observe that

$$
\sqrt{n}\left(\widehat{a}_{n}-a\right)=\frac{\sqrt{n}}{2 \gamma_{n}(0)}\left(\gamma_{n}(1)-\gamma(1)-\frac{\gamma(1)}{\gamma(0)}\left(\gamma_{n}(0)-\gamma(0)\right)\right) .
$$

Hence, we derive from (11) together with Slutsky's lemma that

$$
\sqrt{n}\left(\widehat{a}_{n}-a\right) \stackrel{\mathcal{L}}{\longrightarrow} \mathcal{N}\left(0, \tau^{2}\right)
$$

where

$$
\tau^{2}=36\left(\sigma^{2}(1)+(2 a-1)^{2} \sigma^{2}(0)-2(2 a-1) \sigma(0,1)\right)
$$

\section{Fourier transforms}

In this section, we investigate the asymptotic behavior of Fourier transforms of $\left(\xi_{t}\right)$ given, for all $g \in B V([0,1])$ and all $\theta \in \mathbb{R}$, by

$$
S_{n}(\theta)=\sum_{t=1}^{n} g\left(\xi_{t}\right) e^{i t \theta}
$$

We shall assume that the function $\varphi$ belongs to $B V([0,1])$ and that the expanding map $T$ satisfies (15) and (16). In addition, we suppose that $\mathbb{E}_{\mu}\left[g\left(\xi_{0}\right)\right]=0$ and $\mathbb{E}_{\mu}\left[g^{2}\left(\xi_{0}\right)\right]$ is finite. It is obvious to realize that $S_{n}(\theta)$ shares the same distribution as

$$
\Sigma_{n}(\theta)=\sum_{t=1}^{n} g\left(\varphi\left(Y_{t}\right)\right) e^{i t \theta}
$$

where $\left(Y_{t}\right)$ is the associated Markov chain of the underlying process $\left(X_{t}\right)$. Consequently, we can deduce from [29], which deals with Fourier transforms of stationary and ergodic Markov chains, the asymptotic behavior of $S_{n}(\theta)$. 
Corollary 1 Assume that (15) and (16) are satisfied. Assume moreover that $g$ is in $B V([0,1])$ and satisfies $\mathbb{E}_{\mu}\left[g\left(\xi_{0}\right)\right]=0$ and $\mathbb{E}_{\mu}\left[g^{2}\left(\xi_{0}\right)\right]$ is finite. Then, for almost all $\theta \in \mathbb{R}$, there exists $0 \leq \sigma(\theta)<\infty$ such that

$$
\frac{S_{n}(\theta)}{\sqrt{n}} \stackrel{\mathcal{L}}{\longrightarrow} \mathcal{N}\left(0, \sigma^{2}(\theta) I d_{2}\right)
$$

In addition, for almost all pairs $(\theta, \lambda) \in \mathbb{R}^{2}, S_{n}(\theta) / \sqrt{n}$ and $S_{n}(\lambda) / \sqrt{n}$ are asymptotically independent. Finally, for almost all $\theta \in \mathbb{R}$, the spectral density of the process $\left(g\left(\xi_{t}\right)\right)$ is given by $f_{g}(\theta)=\sigma^{2}(\theta) / \pi$.

Corollary 2 Assume that (15) and (16) are satisfied. Assume moreover that $g$ is in $B V([0,1])$ and satisfies $\mathbb{E}_{\mu}\left[g\left(\xi_{0}\right)\right]=0$ and $\mathbb{E}_{\mu}\left[g^{2}\left(\xi_{0}\right)\right]$ is finite. Then, for all $\theta \in\left[0,2 \pi\left[\right.\right.$ there exists $h_{\theta}\left(\xi_{0}, \xi_{1}\right) \in \mathbb{L}^{2}([0,1])$ such that

$$
\sum_{t=1}^{n} e^{i t \theta}\left(\mathbb{E}\left[g\left(\xi_{t}\right) \mid \xi_{1}\right]-\mathbb{E}\left[g\left(\xi_{t}\right) \mid \xi_{0}\right]\right)
$$

converges in $\mathbb{L}^{2}([0,1])$ to $h_{\theta}\left(\xi_{0}, \xi_{1}\right)$, as $n$ goes to infinity. In addition, we also have $\mathbb{E}\left[\left|\mathbb{E}\left[S_{n}(\theta) \mid \xi_{0}\right]\right|^{2}\right]=o(n)$. Moreover,

(i) if $\theta \neq 0, \pi$,

$$
\frac{S_{n}(\theta)}{\sqrt{n}} \stackrel{\mathcal{L}}{\longrightarrow} \mathcal{N}\left(0, \sigma^{2}(\theta) I d_{2}\right)
$$

where $\sigma^{2}(\theta)=\mathbb{E}\left[h_{\theta}^{2}\left(\xi_{0}, \xi_{1}\right)\right] / 2$;

(ii) if $\theta=0$ or $\pi$

$$
\frac{S_{n}(\theta)}{\sqrt{n}} \stackrel{\mathcal{L}}{\longrightarrow} \mathcal{N}\left(0, \tau^{2}(\theta)\right)
$$

with $\tau^{2}(\theta)=2 \sigma^{2}(\theta)$.

Proof. The proofs of Corollaries 1 and 2 are postponed to Section 8.

Remark. In the last case $\theta=0$ or $\pi$, the result is the central limit theorem stated for example in [6] with limiting variance given by

$$
\tau^{2}(\theta)=\sum_{k=0}^{\infty}<g\left(\varphi\left(T^{k}\right)\right), g(\varphi)>_{\mu}
$$

\section{On the nonstationary case}

We shall now focuse our attention on the asymptotic behavior of the nonstationary process $\left(\xi_{t}^{\prime}\right)$ given, for all $t \in \mathbb{N}$, by

$$
\xi_{t}^{\prime}=\varphi\left(T^{t}\left(X_{0}^{\prime}\right)\right)=\varphi\left(X_{t}^{\prime}\right)
$$


where $T$ is a piecewise expanding map of the interval $[0,1]$ and $\varphi \in B V([0,1])$. The initial state $X_{0}^{\prime}$ is not distributed over $[0,1]$ according to $\mu$ but $X_{0}^{\prime}$ has a probability density function $p \in B V([0,1])$. From a statistical point of view, it is indeed interesting to carry out the asymptotic analysis of functionals which do not depend on the unknown invariant density density function $f_{\mu}$. In many situations, it is possible to prove the convergence of the nonstationary case to the stationary one. To be more precise, let us recall the following inequality $[8,19,28]$ on the Perron-Frobenius operator $\mathcal{L}$ in the case where the dynamical system is generated by a Lasota-Yorke map $T$. One can find some $0<\delta<1$ and $c>0$ such that, for any $n \geq 0$

$$
\left\|\mathcal{L}^{n} p-f_{\mu}\right\|_{\infty} \leq c \delta^{n}
$$

This result allows us to start from any initial state $X_{0}^{\prime}$ with probability density function $p \in B V([0,1])$. Let us explain in details what happens in the case of the periodogram. We know from Section 2 that for any $t \geq 0$

$$
S_{n}(t)=\sqrt{n}\left(\frac{1}{n} \sum_{k=1}^{n-t} \xi_{k} \xi_{k+t}-\gamma(t)\right) \stackrel{\mathcal{L}}{\longrightarrow} \mathcal{N}\left(0, \sigma^{2}(t)\right)
$$

We want to show that

$$
S_{n}^{\prime}(t)=\sqrt{n}\left(\frac{1}{n} \sum_{k=1}^{n-t} \xi_{k}^{\prime} \xi_{k+t}^{\prime}-\gamma(t)\right) \stackrel{\mathcal{L}}{\longrightarrow} \mathcal{N}\left(0, \sigma^{2}(t)\right) .
$$

Denote by $\mathcal{H}$ the set of bounded differentiable functions $h: \mathbb{R} \rightarrow \mathbb{R}$ with continuous and bounded derivative. The set $\mathcal{H}$ is dense in $\mathcal{C}_{b}(\mathbb{R})$. Hence, it is only necessary to prove that, for any $h \in \mathcal{H}$ and $t \geq 0$, the difference

$$
\Delta_{n}(h, t)=\mathbb{E}\left[h\left(S_{n}(t)\right)-h\left(S_{n}^{\prime}(t)\right)\right]
$$

goes to zero as $n$ tends to infinity. Let $h \in \mathcal{H}$ and denote $\theta=\|h\|_{\infty}$ and $\vartheta=\left\|h^{\prime}\right\|_{\infty}$. For any $1 \leq l \leq n-t$, set

$$
S_{n, l}(t)=\frac{1}{\sqrt{n}} \sum_{k=1}^{l} \xi_{k} \xi_{k+t} \quad \text { and } \quad S_{n, l}^{\prime}(t)=\frac{1}{\sqrt{n}} \sum_{k=1}^{l} \xi_{k}^{\prime} \xi_{k+t}^{\prime} .
$$

As $\varphi \in B V([0,1]), \varphi$ is bounded by some constant $M>0$. Hence, both $\left|S_{n, l}(t)\right|$ and $\left|S_{n, l}^{\prime}(t)\right|$ are bounded by $M^{2} l / \sqrt{n}$. Furthermore, if $R_{n, l}(t)=S_{n}(t)-S_{n, l}(t)$ and $R_{n, l}^{\prime}(t)=S_{n}^{\prime}(t)-S_{n, l}^{\prime}(t)$, we can deduce from the mean-value theorem that

$$
\left|h\left(S_{n}(t)\right)-h\left(R_{n, l}(t)\right)\right| \leq \frac{\vartheta M^{2} l}{\sqrt{n}} \quad \text { and } \quad\left|h\left(S_{n}^{\prime}(t)\right)-h\left(R_{n, l}^{\prime}(t)\right)\right| \leq \frac{\vartheta M^{2} l}{\sqrt{n}} .
$$

Consequently, for any $1 \leq l \leq n-t$, we obtain that

$$
\left|\Delta_{n}(h, t)\right| \leq\left|\mathbb{E}\left[h\left(R_{n, l}(t)\right)-h\left(R_{n, l}^{\prime}(t)\right)\right]\right|+\frac{2 \vartheta M^{2} l}{\sqrt{n}} .
$$


However,

$$
\mathbb{E}\left[h\left(R_{n, l}(t)\right)-h\left(R_{n, l}^{\prime}(t)\right)\right]=\int_{0}^{1} h\left(Z_{n, l}(x, t)\right)\left(f_{\mu}(x)-\mathcal{L}^{l} p(x)\right) d x
$$

where

$$
Z_{n, l}(x, t)=\sqrt{n}\left(\frac{1}{n} \sum_{k=1}^{n-t-l} \varphi\left(T^{k}(x)\right) \varphi\left(T^{k+t}(x)\right)-\gamma(t)\right) .
$$

Therefore, we infer from (26) that, for any $1 \leq l \leq n-t$

$$
\left|\Delta_{n}(h, t)\right| \leq \theta c \delta^{l}+\frac{2 \vartheta M^{2} l}{\sqrt{n}}
$$

Finally, as $0<\delta<1$, we can conclude that, for $l$ large enough, $\Delta_{n}(h, t)$ goes to zero as $n$ tends to infinity.

\section{Proofs}

\subsection{Proof of Theorems 1 and 2}

For all $t \in \mathbb{N}$, we immediately deduce from the ergodic theorem that $\gamma_{n}(t)$ converges almost surely to $\gamma(t)$. Then, the strong law (12) clearly follows from Lévy theorem [3, Theorem 2.2. page 106]. The finite dimensional central limit of Theorem 1 is a direct application of [25, Theorem 3 page 58] applied to the reversed process $\left(\varphi\left(Y_{t}\right)\right)$ which is an ergodic strictly stationary sequence with mean zero. Assume that the process $\left(\xi_{t}\right)$ satisfies assumptions (4), (8) and (9). For all $t \in \mathbb{N}$, $\left(X_{0}, X_{1}, \cdots, X_{t}\right)$ shares the same distribution as $\left(Y_{t}, Y_{t-1}, \cdots, Y_{0}\right)$. Consequently, assumptions (4) and (9) hold for the process $\left(\xi_{t}\right)$, they also hold for the reversed process $\left(\varphi\left(Y_{t}\right)\right)$. Moreover assumption (8) yields

$$
\sum_{s=1}^{\infty}\left(\mathbb{E}\left[\left(\mathbb{E}\left[\varphi\left(Y_{s}\right) \varphi\left(Y_{t+s}\right) \mid \varphi\left(Y_{0}\right)\right]-\gamma(t)\right)^{2}\right]\right)^{1 / 2}<+\infty
$$

Hence, assumptions in [25, Theorem 3 page 58] are satisfied for the reversed process $\left(\varphi\left(Y_{t}\right)\right)$, which implies the finite dimensional central limit theorem for $\left(\varphi\left(Y_{t}\right)\right)$ and therefore for $\left(\xi_{t}\right)$. Finally, we complete the proof of Theorem 2 by use of [25, Corollary 2 page 61$]$.

\subsection{Proof of Theorem 3}

First of all, we shall prove that the projective criterion $(a)$ implies that $\gamma$ is finite. For all $t \geq 0$, we have

$$
|\gamma(t)|=\left|\mathbb{E}\left[\xi_{0} \xi_{t}\right]\right|=\left|\mathbb{E}\left[\varphi\left(Y_{0}\right) \varphi\left(Y_{t}\right)\right]\right|
$$


Moreover,

$$
\left|\mathbb{E}\left[\varphi\left(Y_{0}\right) \varphi\left(Y_{t}\right)\right]\right|=\left|\mathbb{E}\left[\varphi\left(Y_{0}\right) \mathbb{E}_{0}\left[\varphi\left(Y_{t}\right)\right]\right]\right| \leq\left\|\varphi\left(Y_{0}\right) \mathbb{E}_{0}\left[\varphi\left(Y_{t}\right)\right]\right\|_{1} \leq \theta_{1,2,1}(t)
$$

Therefore, if $(a)$ holds,

$$
\sum_{t=0}^{\infty}|\gamma(t)| \leq \sum_{t=0}^{\infty} \theta_{1,2,1}(t)<+\infty
$$

which immediately implies that $\gamma$ is finite. Next, let us prove that $(a)$ and $(b)$ lead to $\kappa$ finite. To prove that the sum $\kappa$ of the fourth cumulants of the process $\left(\xi_{t}\right)$ is finite, we prove that the sum $\tau$ of the fourth cumulants of the reversed process $\left(\varphi\left(Y_{t}\right)\right)$ is finite. For this, we shall proceed by induction as in [12] for strong mixing processes. It is necessary to introduce some notations. For $n \geq 1$ and $n$ real valued random variables $A_{1}, \ldots, A_{n}$, define

$$
\operatorname{cum}\left(A_{1}, \ldots, A_{n}\right)=\sum(-1)^{k-1}(k-1) ! \mathbb{E}\left[\Pi_{i \in \nu_{1}} A_{i}\right] \cdots \mathbb{E}\left[\Pi_{i \in \nu_{k}} A_{i}\right]
$$

where $\nu_{1}, \ldots, \nu_{k}$ is a partition of $(1,2, \ldots, n)$ and one sums over all these partitions. Moreover, let $\nu$ be a subset of $\{1, \ldots, n\}$ and define $C_{\nu}=\operatorname{cum}\left(A_{i}, i \in \nu\right)$. We have from [25, Chapter II] that

$$
\mathbb{E}\left[A_{1} \ldots A_{n}\right]=\sum C_{\nu_{1}} \ldots C_{\nu_{k}}
$$

where one sums over all partitions $\nu_{1}, \ldots \nu_{k}$ of $(1,2, \ldots, n)$. As $\left(\varphi\left(Y_{t}\right)\right)$ is centered, we get that for all $(r, s, t) \in \mathbb{N}^{3}, \tau(r, s, t)=\operatorname{cum}\left(\varphi\left(Y_{0}\right), \varphi\left(Y_{r}\right), \varphi\left(Y_{s}\right), \varphi\left(Y_{t}\right)\right)$. For $2 \leq p \leq 4$, let

$$
C_{p}=\sum_{0=t_{1} \leq \ldots \leq t_{p}}\left|c\left(t_{1}, \ldots, t_{p}\right)\right|
$$

where $c\left(t_{1}, \ldots, t_{p}\right)=\operatorname{cum}\left(\varphi\left(Y_{t_{1}}\right), \ldots, \varphi\left(Y_{t_{p}}\right)\right)$. We already saw that

$$
C_{2}=\sum_{t=0}^{\infty}|\gamma(t)|<+\infty
$$

Our purpose is to show by induction that, under $(a)$ and $(b), C_{4}$ is finite. For $p \geq 3$ and $0=t_{1} \leq \ldots \leq t_{p}$, let $r=t_{m+1}-t_{m}$ where

$$
m=\inf \left\{1 \leq m<p / t_{m+1}-t_{m}=\max \left(t_{2}-t_{1}, \ldots, t_{p}-t_{p-1}\right)\right\} .
$$

One can observe that $\left|c\left(t_{1}, \ldots, t_{p}\right)\right|$ is bounded by

$$
\left|\mathbb{E}\left[\varphi\left(Y_{t_{1}}\right) \ldots \varphi\left(Y_{t_{p}}\right)\right]-\mathbb{E}\left[\varphi\left(Y_{t_{1}}\right) \ldots \varphi\left(Y_{t_{m}}\right)\right] \mathbb{E}\left[\varphi\left(Y_{t_{m+1}}\right) \ldots \varphi\left(Y_{t_{p}}\right)\right]\right|+R
$$

with

$$
R \leq \sum_{k \neq 1} \frac{1}{k}\left|\mathbb{E}\left[\Pi_{i \in \nu_{1}} \varphi\left(Y_{i}\right)\right]\right| \ldots\left|\mathbb{E}\left[\Pi_{i \in \nu_{k}} \varphi\left(Y_{i}\right)\right]\right|
$$


where $\nu_{1}, \ldots, \nu_{k}$ is a partition of $\left(t_{1}, \ldots, t_{p}\right)$ with $k \neq 1$. By use of $(30)$, one can find some constant $M(p)>0$ such that

$$
R \leq M(p) \sum_{k \neq 1} \frac{1}{k} \max \left|C_{\nu_{1}} \ldots C_{\nu_{k}}\right|
$$

where the maximum is taken over all partitions $\nu_{1}, \ldots, \nu_{k}$ of $\left(t_{1}, \ldots, t_{p}\right)$ with $k \neq 1$. It follows from (18) that

$$
\left|\mathbb{E}\left[\varphi\left(Y_{t_{1}}\right) \ldots \varphi\left(Y_{t_{p}}\right)\right]-\mathbb{E}\left[\varphi\left(Y_{t_{1}}\right) \ldots \varphi\left(Y_{t_{m}}\right)\right] \mathbb{E}\left[\varphi\left(Y_{t_{m+1}}\right) \ldots \varphi\left(Y_{t_{p}}\right)\right]\right| \leq \theta_{m, p, 1}(r)
$$

Hence, we can deduce from (31) to (33) that

$$
C_{p} \leq \sum_{r=0}^{\infty} r^{p-2} \theta_{m, p, 1}(r)+M(p) \sum_{k \neq 1} \frac{1}{k} \sum_{p_{1}+\ldots+p_{k}=p} C_{p_{1}} \ldots C_{p_{k}} .
$$

The first right hand term is finite because of $(b)$. In addition, the second right hand term is also finite by induction as all $p_{i}<p$. We can conclude that $C_{4}$ and $\tau$ are finite. It remains to prove that $(c)$ implies condition (8). This implication clearly follows from the fact that for all $s, t \geq 0$

$$
\mathbb{E}\left[\xi_{0} \xi_{s} \mid \xi_{t+s}\right]=\mathbb{E}\left[\varphi\left(Y_{s}\right) \varphi\left(Y_{t+s}\right) \mid \varphi\left(Y_{0}\right)\right]
$$

and

$$
\left(\mathbb{E}\left[\left(\mathbb{E}\left[\varphi\left(Y_{s}\right) \varphi\left(Y_{t+s}\right) \mid \varphi\left(Y_{0}\right)\right]-\gamma(t)\right)^{2}\right]\right)^{1 / 2} \leq \theta_{0,2,2}(s)
$$

which completes the proof of Theorem 3.

\subsection{Proof of Theorem 4}

In order to prove Theorem 4, assume that $\varphi \in B V([0,1])$ and let $C=\|D \varphi\|$. Denote by $B V_{1}([0,1])$ the subset of all functions $h \in B V([0,1])$ whose bounded variation norm is smaller than 1 , that is $\|D h\| \leq 1$. As $\varphi$ is bounded by some constant $M>0$, it follows from (18) that for any integers $0 \leq i<j, k \geq 0$ and for any $t=\left(t_{1}, t_{2}, \cdots t_{j}\right) \in \Gamma=\Gamma_{i, j, k}$ and $m \in\{1,2\}$,

$$
\begin{aligned}
\theta_{i, j, m}(k) & =\sup _{t \in \Gamma}\left\|\varphi\left(Y_{t_{1}}\right) \cdots \varphi\left(Y_{t_{i}}\right) \mathbb{E}_{t_{i}}\left[\varphi\left(Y_{t_{i+1}}\right) \cdots \varphi\left(Y_{t_{j}}\right)-\mathbb{E}\left[\varphi\left(Y_{t_{i+1}}\right) \cdots \varphi\left(Y_{t_{j}}\right)\right]\right]\right\|_{m} \\
& \leq \sup _{t \in \Gamma}\left\|\varphi\left(Y_{t_{1}}\right) \cdots \varphi\left(Y_{t_{i}}\right) \mathbb{E}_{t_{i}}\left[\varphi\left(Y_{t_{i+1}}\right) \cdots \varphi\left(Y_{t_{j}}\right)-\mathbb{E}\left[\varphi\left(Y_{t_{i+1}}\right) \cdots \varphi\left(Y_{t_{j}}\right)\right]\right]\right\|_{\infty} \\
& \leq M^{i} \sup _{t \in \Gamma}\left\|\mathbb{E}_{t_{i}}\left[\varphi\left(Y_{t_{i+1}}\right) \cdots \varphi\left(Y_{t_{j}}\right)-\mathbb{E}\left[\varphi\left(Y_{t_{i+1}}\right) \cdots \varphi\left(Y_{t_{j}}\right)\right]\right]\right\|_{\infty} \\
& \leq M^{i} C^{j-i} \Phi_{j-i}(k)
\end{aligned}
$$

with $\mathcal{F}_{t_{i}}=\sigma\left(\varphi\left(Y_{0}\right), \cdots, \varphi\left(Y_{t_{i}}\right)\right) \subset \mathcal{G}_{t_{i}}=\sigma\left(Y_{0}, \cdots, Y_{t_{i}}\right)$

$$
\Phi_{j-i}(k)=\max _{1 \leq l \leq j-i} \sup _{t_{i}+k \leq t_{i+1} \leq \cdots \leq t_{i+l}} \phi\left(\mathcal{G}_{t_{i}}, Y_{t_{i+1}}, \ldots, Y_{t_{i+l}}\right)
$$




$$
\begin{gathered}
\phi\left(\mathcal{G}_{t_{i}}, Y_{t_{i+1}}, \ldots, Y_{t_{i+l}}\right)=\left\|\Delta_{i l}\right\|_{\infty} \\
\Delta_{i l}=\sup \left|\mathbb{E}_{\mathcal{G}_{t_{i}}}\left[\prod_{s=1}^{l}\left(\varphi_{s}\left(Y_{t_{i+s}}\right)-\mathbb{E}\left[\varphi_{s}\left(Y_{t_{i+s}}\right)\right]\right)\right]-\mathbb{E}\left[\prod_{s=1}^{l}\left(\varphi_{s}\left(Y_{t_{i+s}}\right)-\mathbb{E}\left[\varphi_{s}\left(Y_{t_{i+s}}\right)\right]\right)\right]\right|
\end{gathered}
$$

where one takes the supremum over all functions $\varphi_{1}, \ldots, \varphi_{l} \in B V_{1}([0,1])$ and where $\mathbb{E}_{\mathcal{G}_{t_{i}}}$ is the conditional expectation with respect to $\mathcal{G}_{t_{i}}$. Under assumptions (15) and (16), we have for any $h \in B V([0,1])$,

$$
\begin{aligned}
\left\|D K^{n}(h)\right\|=\left\|D K^{n}(h-h(0))\right\| & \leq 2 d\left\|\Psi^{n}\left(f_{\mu}(h-h(0))\right)\right\|_{v}, \\
& \leq 4 \alpha \rho^{n}\|D h\|
\end{aligned}
$$

where $\alpha=2 c d\left(\left\|D f_{\mu}\right\|+1\right)$. Hence, we find using exactly the same lines as $[10$, Lemma 1] that for all $k \geq 0$

$$
\Phi_{3}(k) \leq 2 \alpha\left(1+\beta+\beta^{2}\right) \rho^{k}
$$

with $\beta=4 \alpha$. For any integers $0 \leq i<j$, one can deduce from the definition of $\Phi_{j-i}$ that, for all $k \in \mathbb{N}$,

$$
\Phi_{1}(k) \leq \Phi_{2}(k) \leq \Phi_{3}(k)
$$

Therefore, under assumptions (15) and (16) of Theorem 4, it clearly follows from (34) and (35) that for $0 \leq i<j \leq 4, m \in\{1,2\}$, the coefficients $\theta_{i, j, m}(k)$ decrease exponentially fast to zero as $k$ tends to infinity. Hence the three conditions of Theorem 3 are satisfied, which concludes the proof of Theorem 4.

\subsection{Proof of Corollary 1}

Corollary 1 immediately follows from Theorem 1 and Proposition 2 of [29]. More precisely, we have to check that condition (2) in [29] is satisfied, so that

$$
\sum_{t=1}^{\infty} \frac{1}{t} \mathbb{E}\left[\left(\mathbb{E}\left[g\left(\varphi\left(Y_{t}\right)\right) \mid \varphi\left(Y_{0}\right)\right]\right)^{2}\right]<\infty
$$

For all $t \geq 0$, we have

$$
\mathbb{E}\left[\left(\mathbb{E}\left[g\left(\varphi\left(Y_{t}\right)\right) \mid \varphi\left(Y_{0}\right)\right]\right)^{2}\right] \leq \theta_{0,1,2}^{2}(t)
$$

In addition, under (15) and (16), $\theta_{0,1,2}(t)$ goes exponentially fast to zero as $t$ tends to infinity. Hence, condition (2) in [29] is satisfied. 


\subsection{Proof of Corollary 2}

For $\theta=0, \pi$, Corollary 2 follows from the classical central limit theorem for partial sums. It is stated for example in [6]. For $\theta \neq 0, \pi$, Corollary 2 can be proven by use of Theorem 2 of [29]. More precisely, we have to check that condition (7) in [29] is satisfied, so that

$$
\sum_{t=1}^{\infty}\left\|\mathbb{E}\left[g\left(\varphi\left(Y_{t}\right)\right) \mid \varphi\left(Y_{1}\right)\right]-\mathbb{E}\left[g\left(\varphi\left(Y_{t}\right)\right) \mid \varphi\left(Y_{0}\right)\right]\right\|_{2}<+\infty .
$$

By the triangular inequality, $\left\|\mathbb{E}\left[g\left(\varphi\left(Y_{t}\right)\right) \mid \varphi\left(Y_{1}\right)\right]-\mathbb{E}\left[g\left(\varphi\left(Y_{t}\right)\right) \mid \varphi\left(Y_{0}\right)\right]\right\|_{2}$ is bounded by

$$
\left\|\mathbb{E}\left[g\left(\varphi\left(Y_{t}\right)\right) \mid \varphi\left(Y_{1}\right)\right]\right\|_{2}+\left\|\mathbb{E}\left[g\left(\varphi\left(Y_{t}\right)\right) \mid \varphi\left(Y_{0}\right)\right]\right\|_{2} .
$$

Both terms in (36) decrease exponentially fast to zero as $t$ tends to infinity, which concludes the proof of Corollary 2.

Aknowledgments. The authors are very grateful to J. R. León for many fruitful discussions.

\section{References}

[1] H. D. I. Abarbanel, R. Brown, J.J. Sidorowich and L. S. Tsimring, (1993). The analysis of observed chaotic data in physical systems. Rev. Modern Phys. 65, no. 4, 1331-1392.

[2] V. Ananthram, (2004). Queuing analysis with traffic models based on deterministic dynamical systems. Comput. Sci. J. Moldova 12, no. 2, 154-170.

[3] R. Azencott, D. Dacunha-Castelle. Séries d'observations irrégulières. Masson, 1984.

[4] A. D. Barbour, R. M. Gerrard and G. Reinert, (2000). Iterates of expanding maps. Probab. Theory Relat. Fields 116 151-180.

[5] J. M. Bardet, P. Doukhan and J. R. León, (2005). Uniform limit theorems for the periodogram of weakly dependent time series and their applications to Whittle's estimate. http://samos .univ-paris1.fr/.

[6] A. Broise, (1996). Transformations dilatantes de l'intervalle et théorèmes limites. Études spectrales d'opérateurs de transfert et applications. Astérisque 238 1-109.

[7] J.-R. Chazottes, P. Collet and B. Schmitt, (2005). Statistical consequences of Devroye inequality for processes. Applications to a class of non-uniformly hyperbolic dynamical systems. Nonlinearity. 18 2341-2364.

[8] P. Collet, (1996). Some ergodic properties of maps of the interval. Dynamical systems (Temuco, 1991/1992), 55-91, Travaux en cours, 52, Hermann, Paris.

[9] J. Dedecker and C. Prieur, (2005). New dependence coefficients. Examples and applications to statistics. Probab. Theory and Relat. Fields. 132 203-236.

[10] J. Dedecker and C. Prieur, (2005). An empirical central limit theorem for dependent sequences. Preprint $\mathbf{9}$.

http://www.lsp.ups-tlse.fr/Recherche/Publications/2005/ded09.html 
[11] J. Dedecker and E. Rio, (2005). On Esseen's mean central limit theorem for dependent sequences. Preprint 138.

http://www.math.uvsq.fr/laboratoire/activites/dossierprepub/index.html

[12] P. Doukhan and J. R. León, (1989). Cumulants for stationary mixing random sequences and applications to empirical spectral density. Probab. Math. Stat. 10 11-26.

[13] P. Doukhan and S. Louhichi (1999). A new weak dependence condition and applications to moment inequalities. Stochastic Process. Appl. 84 313-342.

[14] R.M. Dudley (1989). Real analysis and probability. Wadworsth Inc., Belmont, California.

[15] A. Erramilli, R. P. Singh and P. Pruthi (1995). An application of deterministic chaotic maps to model packet traffic. Queueing Systems Theory Appl. 20, no. 1-2, 171-206.

[16] M. Fréchet (1957). Sur la distance de deux lois de probabilité. C.R. Acad. Sci. Paris. 244, 689-692.

[17] M. Giampieri and S. Isola, (2005). A one-parameter family of analytic markov maps with an intermittency transition. Discrete and Continuous Dyn. Syst. 12 115-136.

[18] A. Lasota and J. A. Yorke (1974). On the existence of invariant measures for piecewise monotonic transformations. Trans. Amer. Math. Soc. 186 481-488.

[19] C. Liverani, (1995). Decay of correlations for piecewise expanding maps. J. Statist. Phys. 78, no. 3-4, 1111-1129.

[20] C. Liverani, B. Saussol, S. Vaienti, (1998). Conformal measure and decay of correlation for covering weighted systems. Ergodic Theory Dynam. Systems 18 no. 6, 1399-1420.

[21] A.O. Lopes and S.R.C. Lopes (2002). Convergence in distribution of the periodogram of chaotic processes. Stoch. Dyn. 2, no. 4, 609-624.

[22] A.O. Lopes and S.R.C. Lopes (1998). Parametric estimation and spectral analysis of chaotic time series. Adv. in Applied Probability 30 no. 3, 757-776.

[23] T. Morita, (1994). Local limit theorem and distribution of periodic orbits of LasotaYorke transformations with infinite Markov partition. J. Math. Soc. Japan 46 309-343.

[24] C. Prieur, (2001). Density estimation for one-dimensional dynamical systems. ESAIM Probab. Statist. 5, 51-76.

[25] M. Rosenblatt, (1985). Stationary sequences and random fields. Birkhäuser, Boston.

[26] W. M. Schmidt, (1980). Diophantine approximation. Lectures Notes in Mathematics 785.

[27] Y. Takahashi. $\beta$-transformations and symbolic dynamics. Proceedings of the Second Japan-USSR Symposium on Probability Theory (Kyoto, 1972), 455-464. Lecture Notes in Math., Vol. 330, Springer, Berlin (1973).

[28] M. Viana, (1997). Stochastic dynamics of deterministic systems, Instituto de Matematica Pura e Aplicada. IMPA, 21.

[29] W. B. Wu, (2005). Fourier transforms of stationary processes. Proc. Amer. Math. Soc. 133, no. 1, 285-293. 